\title{
Regulation of pyrBI operon expression in Escherichia coli by UTP-sensitive reiterative RNA synthesis during transcriptional initiation
}

\author{
Chongguang Liu, Lucie S. Heath, and Charles L. Turnbough Jr. ${ }^{1}$ \\ Department of Microbiology, University of Alabama at Birmingham, Birmingham, Alabama 35294 USA
}

\begin{abstract}
Pyrimidine-mediated regulation of pyrBI operon expression in Escherichia coli K-12 occurs through UTP-sensitive transcriptional attenuation and through a second mechanism that functions at the level of transcriptional initiation. In this study we demonstrate that this second control mechanism is based on UTP-sensitive reiterative RNA synthesis within a run of three T-A base pairs in the pyrBI initially transcribed region. Our results show that high UTP levels induce the synthesis in vitro of nascent transcripts with the sequence AAUUUU ${ }_{n}$ (where $n=1$ to $>30$ ), which are not extended downstream to include pyrBI sequences. Synthesis of these transcripts, which are initiated at the predominant in vivo transcriptional start site, inhibits the production of full-length pyrBI transcripts. A TTT to GTA mutation in the pyrBI initially transcribed region eliminates reiterative transcription and stimulates productive transcription in vitro. When introduced into the $E$. coli chromosome, this mutation causes a sevenfold increase in pyrBI expression in cells grown under conditions of pyrimidine excess and nearly abolishes pyrimidine-mediated regulation of pyrBI expression when coupled with a mutation that eliminates attenuation control. Additional experiments indicate that the context of the three T-A base pairs within the pyrBI initially transcribed region is important for reiterative transcription. A possible mechanism for reiterative transcription and the likely involvement of this process in the regulation of other genes are discussed.
\end{abstract}

[Key Words: Pyrimidine gene expression; transcriptional slippage; pseudotemplated transcription; RNA polymerase stuttering; promoter clearance]

Received June 27, 1994; revised version accepted October 5, 1994.

In Escherichia coli $\mathrm{K}-12$, the pyrBI operon encodes the two nonidentical subunits of the pyrimidine biosynthetic enzyme aspartate transcarbamylase (ATCase). Expression of the pyrBI operon is regulated over an $\sim 300$ fold range by pyrimidine availability. Most of this regulation (i.e., 50-fold) occurs through a UTP-sensitive attenuation control mechanism in which low intracellular levels of UTP cause transcriptional pausing within a segment of the pyrBI leader region that specifies a 44codon open reading frame. This pausing permits close coupling of transcription and translation within the leader region, which suppresses transcriptional termination at an attenuator (a $\rho$-independent transcriptional terminator) located ${ }_{1}$ in front of the $p_{y r} B$ structural gene (Turnbough et al. 1983; Roland et al. 1988; Liu and Turnbough 1989; Landick and Turnbough 1992; Donahue and Turnbough 1994).

Pyrimidine-mediated regulation of $\mathrm{pyr} B I$ expression also occurs at the level of transcriptional initiation (Don-

${ }^{1}$ Corresponding author. ahue and Turnbough 1990; Liu et al. 1993). Recent studies have suggested that this regulation involves a run of three T-A base pairs (specifying UUU) in the initially transcribed region of the pyrBI promoter (Donahue and Turnbough 1990). The pyrBI promoter region contains the sequence 5'-TATAATGCCGGACAATTTGCCG; the -10 region and two adenine residues at which transcriptional initiation can occur in vitro are underlined, and the more downstream adenine residue appears to be the predominant transcriptional start site in vivo (Donahue and Turnbough 1990). It was discovered that RNA polymerase formed heparin-resistant, transcriptionally competent initiation complexes at the pyr $B I$ promoter in the presence of ATP and CTP and, to a small extent, with ATP alone, indicating stabilization by the nascent transcripts ACAA and AA, respectively. In contrast, in the presence of ATP and UTP, RNA polymerase did not form initiation complexes that were capable of synthesizing $p y r B I$ transcripts following a heparin challenge. This result suggested that the synthesis of a nascent transcript with the sequence AAUUU did not stabilize the initiation complex or perhaps interfered with pro- 
moter clearance. It was proposed that this effect could be modulated by the intracellular concentration of UTP and contribute to pyrimidine-mediated regulation (Donahue and Turnbough 1990).

In this study we examined the effects of UTP concentration on the initial phases of pyrBI transcription. Characterization of transcripts synthesized in vitro showed that high UTP levels induce reiterative RNA synthesis during transcription of the run of three $\mathrm{T}$-A base pairs in the initially transcribed region of the pyrBI promoter. This process generates transcripts containing long runs of uridine residues that cannot be extended into productive pyr $B I$ transcripts. We also determined the regulatory effects of a mutation that eliminates reiterative transcription in the pyrBI initially transcribed region. The results indicate that UTP-induced reiterative transcription at the pyrBI promoter accounts for nearly all attenuation-independent, pyrimidine-mediated regulation of pyrBI expression. Additional experiments are included that permit an initial characterization of the reiterative transcription reaction.

\section{Results}

UTP-induced reiterative RNA synthesis within the pyrBI initially transcribed region

The effects of UTP concentration on pyrBI promoter clearance were examined by in vitro transcription of the pyr $B I$ promoter-leader region in reaction mixtures containing $200 \mu \mathrm{M}$ each $\left[\gamma^{-32} \mathrm{P}\right] \mathrm{ATP}, \mathrm{CTP}$, and GTP and either 20 or $200 \mu \mathrm{M}$ UTP. $\left[\gamma^{-32} \mathrm{P}\right] \mathrm{ATP}$ was included to label pyrBI transcripts at their $5^{\prime}$ ends. Reaction products were separated by electrophoresis in a $25 \%$ polyacrylamide gel to detect short transcripts. An autoradiograph of the gel revealed an unusual ladder of transcripts generated at $200 \mu \mathrm{M}$ UTP, with the longest transcript in the ladder containing well over 30 nucleotides (Fig. 1, lane 2). Synthesis of this ladder was significantly reduced at $20 \mu \mathrm{M}$ UTP (Fig. 1, lane 1). To tentatively identify these transcripts, transcription reactions were performed with selected nucleoside triphosphates. With $200 \mu \mathrm{M}\left[\gamma^{32} \mathrm{P}\right]-$ ATP alone, no transcripts were synthesized (Fig. 1, lane 3). With $200 \mu \mathrm{M}\left[\gamma^{-32}\right.$ P]ATP plus $200 \mu \mathrm{M}$ UTP, a prominent ladder was produced (Fig. 1, lane 5). Synthesis of the ladder was greatly diminished when the UTP concentration was reduced to $20 \mu \mathrm{M}$ UTP (Fig. 1, lane 4). The level of ladder transcript synthesis was severalfold higher in reactions containing ATP and UTP compared with that in reactions containing all four nucleoside triphosphates (cf. lane 1 with lane 4 and lane 2 with lane 5). These results indicated that the ladder consisted of transcripts with the sequence $\mathrm{AAUUUU}_{n}$ (where $n=1$ to $>30$ ), which apparently were produced by reiterative RNA synthesis within the run of three T-A base pairs (AAA in the template strand) of the pyrBI initially transcribed region. The inclusion of $200 \mu \mathrm{M}$ GTP in the transcription reaction mixture, along with $\left[\gamma^{-32}\right.$ P]ATP and UTP, had little effect on ladder synthesis, except that it produced one

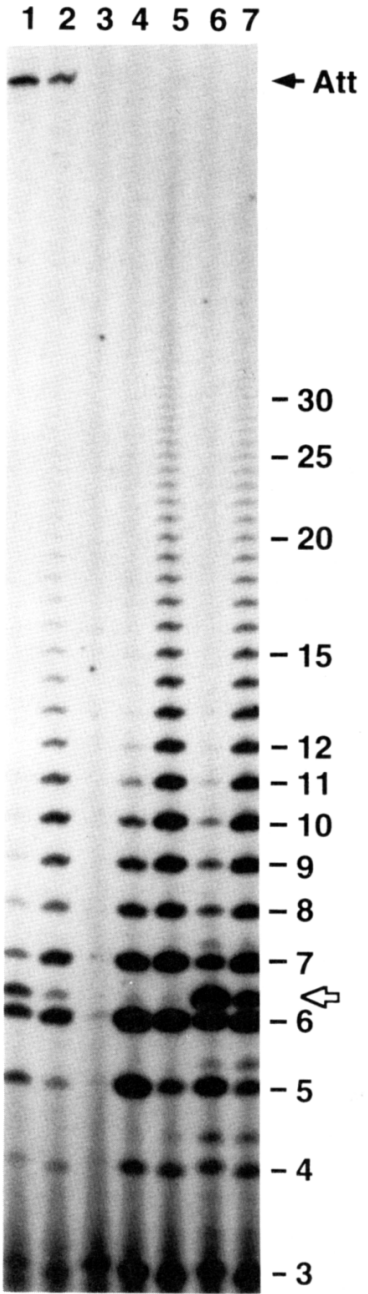

Figure 1. Nucleotide requirements for reiterative RNA synthesis within the pyrBI initially transcribed region. The pyrBI promoter-leader region was transcribed in vitro in reaction mixtures containing the following nucleoside triphosphates: $200 \mu \mathrm{M}$ each $\left[\gamma^{-32} \mathrm{P}\right] \mathrm{ATP}, \mathrm{GTP}$, and CTP and $20 \mu \mathrm{M}$ UTP (lane 1); $200 \mu \mathrm{M}$ each $\left[\gamma^{-32} \mathrm{P}\right] \mathrm{ATP}, \mathrm{GTP}, \mathrm{CTP}$, and UTP (lane 2); $200 \mu \mathrm{M}$ $\left[\gamma^{-32} \mathrm{P}\right]$ ATP (lane 3); $200 \mu \mathrm{M}\left[\gamma^{-32} \mathrm{P}\right] \mathrm{ATP}$ and $20 \mu \mathrm{M}$ UTP (lane 4); $200 \mu \mathrm{M}$ each $\left[\gamma^{32} \mathrm{P}\right]$ ATP and UTP (lane 5); $200 \mu \mathrm{M}$ each $\left[\gamma_{-}^{-32}\right.$ P]ATP and GTP and $20 \mu \mathrm{M}$ UTP (lane 6); $200 \mu \mathrm{M}$ each $\left[\gamma^{-32} \mathrm{P}\right] \mathrm{ATP}, \mathrm{GTP}$, and UTP (lane 7). An autoradiograph is shown of a $25 \%$ polyacrylamide gel used to separate the transcripts. Transcript lengths (in nucleotides) were determined by use of a sizing ladder generated by partial alkaline hydrolysis of purified $\left[\gamma^{32} \mathrm{P}\right] \mathrm{ATP}-\mathrm{labeled}$ pyrBI transcripts terminated at the attenuator (Donahue and Turnbough 1994). Bands corresponding to attenuated pyrBI transcripts (Att), which are 132-135 nucleotides long, and to the aborted transcript AAUUUG (open arrow) are indicated. Termination efficiency at the pyrBI attenuator is $\sim 98 \%$.

additional major transcript (and several new minor transcripts). This major transcript ran slightly slower than the 6-mer synthesized with ATP and UTP alone, and it was presumed to be an aborted transcript with the sequence AAUUUG (Fig. 1, lanes 6,7). Apparently, this 
aborted transcript also was synthesized in reaction mixtures containing all four nucleoside triphosphates (Fig. 1, lanes 1,2 ). The effects of UTP concentration on the synthesis of $p y r B I$ transcripts terminated at the attenuator (i.e., attenuated transcripts) will be described below.

Additional evidence supporting the proposed sequence assignments for the ladder transcripts was provided by performance of three parallel in vitro transcription reactions with $200 \mu \mathrm{M}$ each ATP, CTP, GTP, and UTP /data not shown). In these reactions, the radiolabeled nucleotide was either $\left[\gamma_{-}{ }^{32} \mathrm{P}\right] \mathrm{ATP},\left[\alpha^{-32} \mathrm{P}\right] \mathrm{ATP}$, or $\left[\alpha^{-32} \mathrm{P}\right] \mathrm{UTP}$. Transcripts were separated as described for Figure 1, and the amount of radioactive label was measured in each transcript from 5 to 13 nucleotides long lexcluding the presumed AAUUUG transcript, which was labeled in all reactions|. For each transcript, the molar ratio of $\left[\alpha{ }^{-32} \mathrm{P}\right]$ ATP to $\left[\gamma^{-32} \mathrm{P}\right] \mathrm{ATP}$ was a constant 2 to 1 , indicating that each transcript contained two adenine residues. In contrast, the molar ratio of $\left[\alpha{ }^{-32} \mathrm{P}\right] \mathrm{UTP}$ to $\left[\gamma-{ }^{32} \mathrm{P}\right] \mathrm{ATP}$ increased with transcript length. This ratio was $n-2$ to 1 , where $n$ is the transcript length in nucleotides, indicating that the remaining nonadenine residues in the ladder transcripts were uridines. In addition to these experiments, similar transcriptions were done with $200 \mu \mathrm{M}$ of each nucleoside triphosphate and either $\left[\alpha^{-32} \mathrm{P} \mid \mathrm{GTP}\right.$ or $\left[\alpha^{-32} \mathrm{P}\right]$ CTP as the label (data not shown). With $\left[\alpha-{ }^{32} \mathrm{P}\right] \mathrm{GTP}$, the only ladder transcript labeled was the presumed AAUUUG transcript, indicating that it was identified correctly. With $\left[\alpha-{ }^{32} \mathrm{P}\right] \mathrm{CTP}$, no ladder transcripts were labeled, indicating that none of these transcripts were initiated at the more upstream adenine transcriptional start site, which would have introduced a cytidine residue in the second position of the transcripts.

It also is noteworthy that the pattern of UTP-induced ladder transcript synthesis was bimodal when expressed

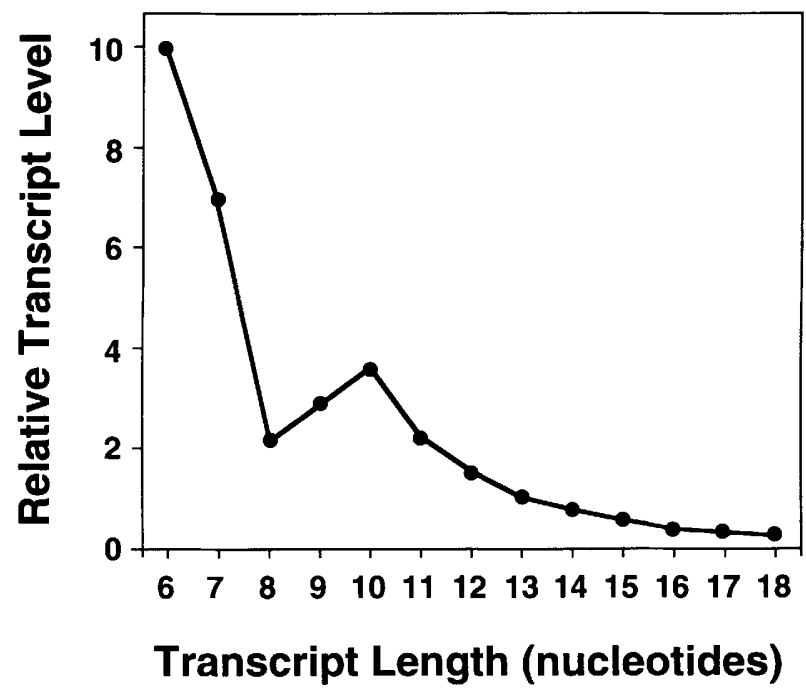

Figure 2. Bimodal pattern of UTP-induced ladder transcript synthesis. Relative levels of the AAUUUU ${ }_{n}$ transcripts from 6 to 18 nucleotides long shown in Fig. 1, lane 2, were measured with a PhosphorImager and plotted against transcript length. as transcript level versus transcript length (Fig. 2). This pattern indicates that the probability of release of a transcript after the addition of the next uridine residue is not constant, at least for transcripts shorter than 10 nucleotides. This pattern is unlike that expected for simple abortive initiation and apparently reflects selective synthesis of particular short ladder transcripts.

\section{Regulatory effects of a pyrBI promoter mutation that eliminates reiterative transcription}

To examine the role of UTP-sensitive reiterative transcription in pyrimidine-mediated regulation of pyrBI expression, we constructed an oligonucleotide-directed mutation, designated pyrBp488, which introduces a TTT to GTA substitution (nontemplate strand sequences) in the pyr $B I$ initially transcribed region. This mutation was shown to eliminate UTP-induced reiterative transcription within the pyr $B I$ initially transcribed region in vitro (Fig. 3, lane 3). In addition, this mutation caused a 3.3fold increase in the synthesis of attenuated pyrBI transcripts (Fig. 3, cf. lanes 1 and 3). By allelic replacement, the pyrBp488 mutation was incorporated into the chromosomal pyrBI operons of strains CLT42 (pyrBI ${ }^{+}$car-94) and CLT71 ( $\triangle$ pyrBa483 car-94) to generate strains CLT214 and CLT235, respectively. These strains are pyrimidine auxotrophs as a result of the car-94 mutation, which inactivates the first enzyme in the pyrimidine biosynthetic pathway. The $\triangle p y r B a 483$ mutation carried by strains CLT71 and CLT235 is a 9-bp deletion that removes the run of eight T-A base pairs at the end of the pyrBI attenuator, which abolishes transcriptional termination activity at this site (Liu and Turnbough 1989).

The effects of the pyrBp488 (TTT to GTA) mutation on pyrBI expression and regulation were determined by measurement of ATCase levels in strains CLT42, CLT71, CLT214, and CLT235 that were grown in minimal medium containing either uracil or UMP as the sole pyrimidine source (Table 1). Growth on uracil provides a condition of pyrimidine excess, and growth on UMP, which is only slowly metabolized by the cells, results in pyrimidine limitation. The results show that the pyrBp 488 mutation caused a sevenfold increase in pyrBI expression under conditions of pyrimidine excess [cf. strains CLT42 (pyrBI ${ }^{+}$) and CLT214 (pyrBp488) on uracil] and that this effect was independent of the attenuator [cf. strains CLT71 ( $\triangle$ pyrBa483) and CLT235 (pyrBp488 $\Delta$ pyrBa483) on uracil]. The pyrBp488 mutation also caused an eightfold reduction in the range of regulation measured with strain CLT214 and nearly eliminated regulation when combined with the attenuator deletion in strain CLT235. The pyrBp488 mutation did not cause large changes in pyrBI expression under conditions of pyrimidine limitation, indicating that the mutation did not significantly alter promoter strength or pyrBI transcript stability. Together, these results indicate that UTP-induced reiterative RNA synthesis accounts for nearly all attenuation-independent pyrimidine-mediated regulation of $p y r B I$ expression. 


\section{3}

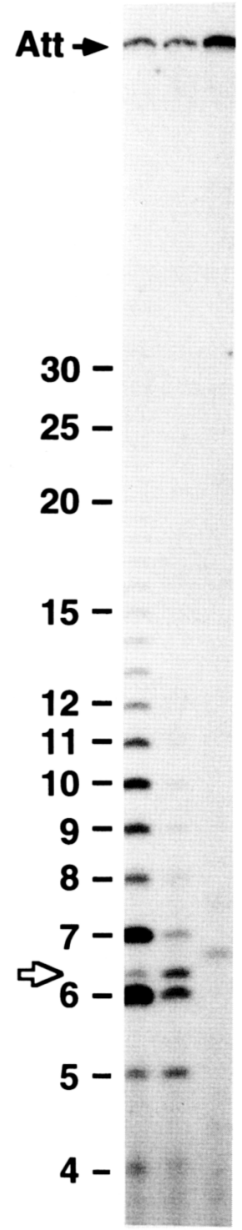

Figure 3. Effects of the pyrBp 488 mutation on reiterative and productive transcription from the pyrBI promoter. Standard in vitro transcription reactions (200 $\mu \mathrm{M}$ NTPs) with $\left[\gamma^{-}{ }^{32} \mathrm{P}\right] \mathrm{ATP}$ as the label were performed with the indicated DNA templates and modifications: wild-type template (lane 1); wild-type template and $20 \mu \mathrm{M}$ UTP (lane 2); and template containing the pyrBp488 mutation (lane 3 ). Transcripts were analyzed as described in the legend to Fig. 1.

UTP concentration dependence and time course of reiterative and productive transcription from the pyrBI promoter in vitro

The concentration of UTP in E. coli varies from $\sim 50-100$ $\mu \mathrm{M}$ in cells limited for pyrimidines to $1 \mathrm{mM}$ in cells grown under conditions of pyrimidine excess (Turnbough 1983; Neuhard and Nygaard 1987; Andersen et al. 1991) To measure the level of reiterative transcription at different physiological concentrations of UTP, standard in vitro transcription reactions were performed in which the UTP concentration was varied from 20 to $1000 \mu \mathrm{M}$. The amount of total ladder transcript synthesis (excluding the aborted transcript AAUUUG) increased almost linearly from 20 to $600 \mu \mathrm{M}$, and then increased much more slowly until it appeared to be near optimal at 1000 $\mu \mathrm{M}$ (Fig. 4A). Levels of individual ladder transcripts increased in parallel over an approximately sevenfold range, with half-maximal synthesis occurring at $\sim 300$ $\mu M$ UTP. In contrast, levels of the aborted transcript AAUUUG decreased over a similar range with increasing UTP concentration.

To examine the effects of UTP concentration on the synthesis of attenuated pyr $B I$ transcripts initiated at the second adenine start site, the start site for ladder transcript synthesis, it was necessary to separate these transcripts from those initiated at the first adenine start site. This separation was accomplished, at least in part, by electrophoresis in an $8 \%$ polyacrylamide gel (Fig. 4B). At $20 \mu \mathrm{M}$ UTP, $76 \%$ of the attenuated pyrBI transcripts are initiated at the second adenine start site (see below), and these transcripts are predominantly 132 nucleotides long. Transcripts initiated at the first adenine start site are predominantly 134 nucleotides long. The lengths of the transcripts initiated at the two start sites gradually increase by one nucleotide with increasing UTP concentration because of a shift in the position of transcriptional termination at the $p y r B I$ attenuator (Donahue and Turnbough 1994). A comparison of the levels of transcripts initiated at the second adenine start site (132nucleotide-long transcripts at $20 \mu \mathrm{M}$ UTP and 133-nucleotide-long transcripts at $1000 \mu \mathrm{M}$ UTP) showed that transcript synthesis was reduced over a greater than fivefold range with increasing UTP concentrations. The size of this effect is probably underestimated, because the band containing the 133-nucleotide-long transcripts also contains comigrating 134-nucleotide-long transcripts initiated at the first adenine start site. Increasing UTP concentrations appeared to have a slight stimulatory effect on the synthesis of attenuated transcripts initiated at the first adenine start site (Fig. 4B, cf. 134-nucleotide-long transcripts at $20 \mu \mathrm{M}$ UTP and 135-nucleotide-long transcripts at $1000 \mu \mathrm{M}$ UTP).

The effects of reaction time on ladder transcript synthesis were examined by performance of a scaled-up standard transcription reaction from which samples were removed after incubation times ranging from 1 to $30 \mathrm{~min}$. Transcription was terminated immediately in

Table 1. Effect of the pyrBp488 (TTT to GTA) mutation on ATCase activity

\begin{tabular}{|c|c|c|c|}
\hline \multirow{2}{*}{$\begin{array}{l}\text { Strain } \\
\text { (pyrBI genotype })\end{array}$} & \multicolumn{2}{|c|}{$\begin{array}{c}\text { ATCase activity } \\
(\mathrm{nmole} / \mathrm{min} \text { per } \mathrm{mg})^{\mathrm{a}}\end{array}$} & \multirow{2}{*}{$\begin{array}{l}\text { Fold } \\
\text { regulation }\end{array}$} \\
\hline & uracil & UMP & \\
\hline CLT42 (pyrBI $\left.{ }^{+}\right)$ & 26.4 & 8,150 & 309 \\
\hline CLT214 (pyrBp488) & 184 & 6,800 & 37 \\
\hline CLT71 (ApyrBa483) & 1,270 & 8,830 & 7.0 \\
\hline \multicolumn{4}{|l|}{ CLT235 (pyrBp488 } \\
\hline$\Delta p y r B a 483 \mid$ & 7,310 & 12,500 & 1.7 \\
\hline
\end{tabular}

Doubling times were $48 \pm 1 \mathrm{~min}$ for all strains grown on uracil, $64 \pm 1 \mathrm{~min}$ for strains CLT42 and CLT214 grown on UMP, and $69 \pm 2 \mathrm{~min}$ for strains CLT71 and CLT235 grown on UMP.

${ }^{a}$ Mean of two experiments with variation of $<3 \%$. 


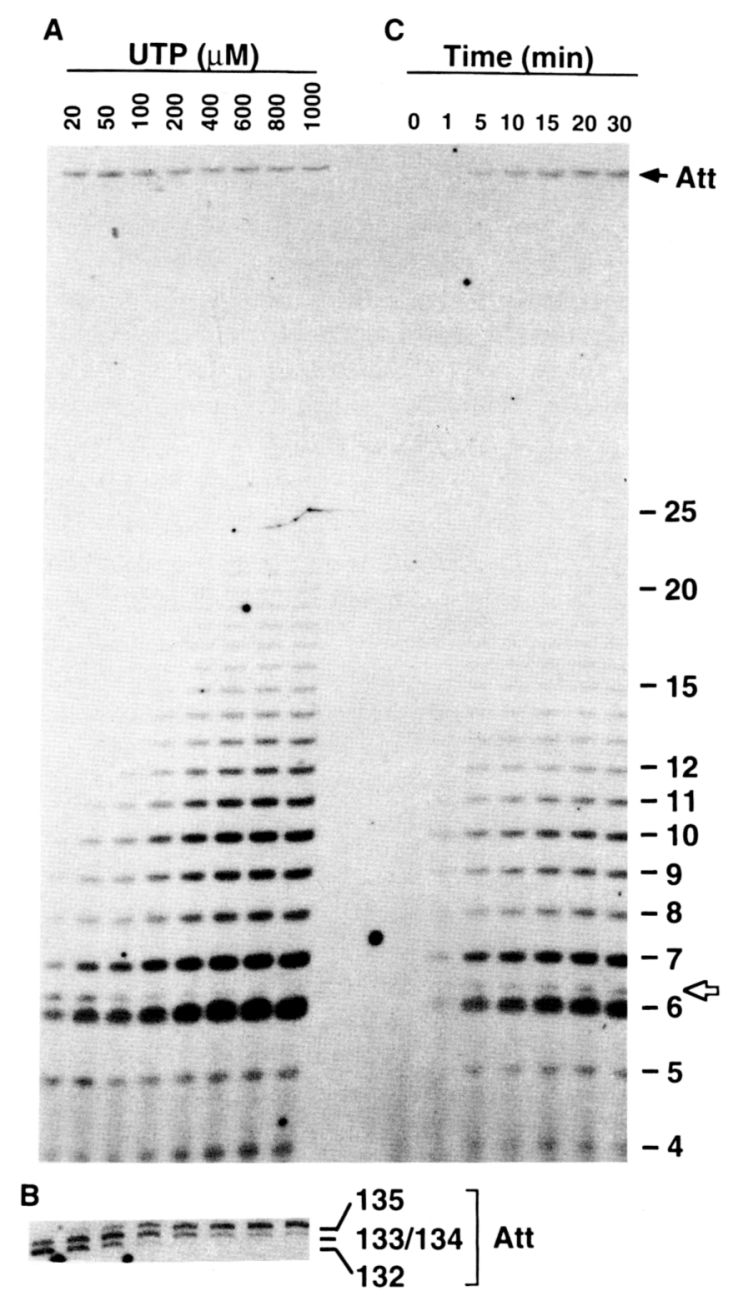

Figure 4. Effects of UTP concentration and reaction time on reiterative and productive transcription from the pyrBI promoter. Standard in vitro transcription reactions with $\left[\gamma^{-}{ }^{32} \mathrm{P}\right] \mathrm{ATP}$ as the label were performed in which the UTP concentration $\mid A, B)$ or incubation time $(C)$ was varied as indicated. Transcripts were separated by electrophoresis in a $25 \%(A, C)$ or an $8 \%(B)$ polyacrylamide gel. In the $8 \%$ gel, the 133 - and 134-nucleotidelong attenuated transcripts initiated at the second and first adenine start sites, respectively, comigrate (Liu and Turnbough 1989|. Other details are as described in the legend to Fig. 1.

each sample, and then the transcripts were analyzed. The results showed that ladder transcript synthesis increased nearly linearly for $\sim 10 \mathrm{~min}$ and then gradually reached a plateau (as a result of inactivation of RNA polymerase) (Fig. 4C). At all times, levels of individual ladder transcripts increased in parallel. It did not appear that longer incubation times allowed short transcripts to be chased into longer ladder transcripts or into attenuated pyrBI transcripts. Results essentially identical to those in Figure $4 \mathrm{C}$ were obtained when the transcription reaction was initiated by the addition of nucleoside triphosphates instead of RNA polymerase or when heparin $(0.1 \mathrm{mg} / \mathrm{ml})$ was added after the indicated incubation times, followed by an additional 10-min incubation.
The latter result indicates that reiterative transcription is inhibited by heparin.

\section{Evidence that reiterative transcription at the pyrBI promoter is a nonproductive process}

To determine directly whether the ladder transcripts could be extended to include downstream pyrBI sequences, ${ }^{32} \mathrm{P}-5^{\prime}$-end-labeled attenuated pyr $B I$ transcripts synthesized under standard transcription conditions with either 20 or $200 \mu \mathrm{M}$ UTP were purified and digested with RNase $T_{1}$. RNase $T_{1}$ cleaves the phosphodiester bond of 5'-GpN, leaving a 3' phosphate. The radiolabeled oligonucleotides produced by the digestion were separated by gel electrophoresis and visualized by autoradiography (Fig. 5, lanes 2,3). Only two major bands migrating at positions indicative of a 6 -mer and an 8-mer were detected (the faint bands were due to incomplete digestion). These bands correspond to the 5 ' oligomers of the transcripts initiated at the two adenine start sites of the pyrBI promoter (i.e., ACAAUUUG and AAUUUG). The AAUUUG band accounted for $76 \%$ and $64 \%$ of total oligonucleotides at 20 and $200 \mu \mathrm{M}$ UTP, respectively. The absence of a ladder of RNase $\mathrm{T}_{1}$-generated oligonucleotides indicates that attenuated transcripts were not derived from ladder transcripts. In a control experiment, ${ }^{32} \mathrm{P}-5^{5}$-end-labeled attenuated pyrBI transcripts synthesized from a mutant template containing a TTT to AAA (nontemplate strand sequences) substitution ( $p y r B p 489$ ) in the pyr $B I$ initially transcribed region were purified. With the mutant template, a low-level of ATP-induced reiterative transcription at the $p y r B I$ promoter can be detected, and attenuated transcripts synthesized with this template migrate as a broad band during gel electrophoresis. Digestion of these attenuated transcripts with RNase $T_{1}$ produced a ladder of ${ }^{32} \mathrm{P}$-labeled oligonucleotides, indicating that in this case ladder transcripts could be extended to include downstream pyrBI sequences (Fig. 5, lane 4).

\section{Discussion}

The results of this study indicate that pyrimidine-mediated, attenuation-independent regulation of pyrBI expression occurs through UTP-sensitive reiterative RNA synthesis during transcriptional initiation. This process is directed by three T-A base pairs in the pyrBI initially transcribed region and produces nascent transcripts with the sequence AAUUUU ${ }_{n}$. These transcripts are not extended downstream to include pyrBI sequences, and their synthesis inhibits the production of full-length pyrBI transcripts initiated at the predominant in vivo transcriptional start site (additional evidence for the latter effect was recently published in Jin and Turnbough 1994). Reiterative transcription in vitro is maximal at UTP concentrations comparable to those found in wildtype $E$. coli or in pyrimidine auxotrophs grown on uracil and is strongly suppressed at low UTP concentrations similar to those found in cells limited for pyrimidines. A mutation in the pyrBI initially transcribed region 


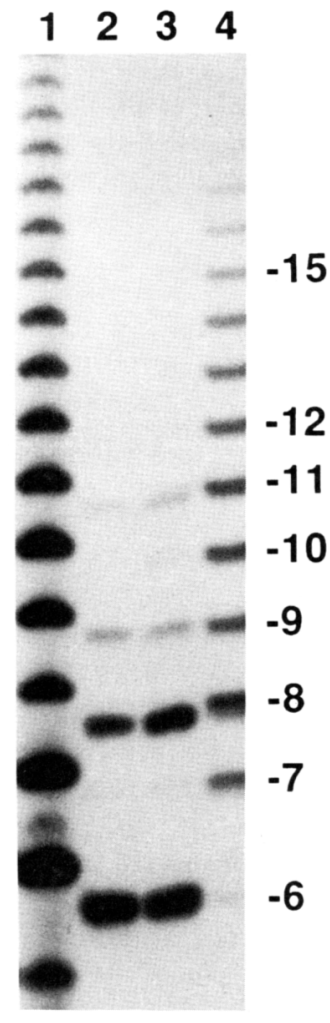

Figure 5. RNase $\mathrm{T}_{1}$ cleavage of purified, ${ }^{32} \mathrm{P}-5^{\prime}$-end-labeled attenuated pyrBI transcripts. Standard in vitro transcription reactions with $\left[\gamma^{32}\right.$ P $]$ ATP as the label were performed with the indicated DNA templates and modifications: wild-type template and $20 \mu \mathrm{M}$ UTP (lane 2); wild-type template (lane 3); and template containing the pyrBp489 (TTT $\rightarrow$ AAA) mutation (lane 4). Attenuated pyrBI transcripts $(\sim 135$ nucleotides long) were purified and digested with RNase $T_{1}$ as described previously (Donahue and Turnbough 1994). Digestion products were analyzed as described in the legend to Fig. 1. Lane 1 shows the UTP-induced ladder synthesized under the standard conditions described for lane 1 in Fig. 3 and is included for size markers. The ladder in lane 1 begins at the bottom with the 5-mer AAUUU. The RNase $T_{1}$-generated oligonucleotides, which have 3 ' phosphates, migrate slightly faster than ladder transcripts containing the same number of nucleotides.

(pyrBp488) that eliminates reiterative transcription, stimulates attenuated $p y r B I$ transcript synthesis in vitro. In $E$. coli, this mutation results in a sevenfold increase in pyr $B I$ expression under conditions of pyrimidine excess and nearly eliminates pyrimidine-mediated regulation of operon expression when coupled with a mutation that inactivates the pyr $B I$ attenuator.

Reiterative transcription /also referred to as pseudotemplated transcription, transcriptional slippage, and RNA polymerase stuttering/ is a well-documented property of RNA polymerase, which was first described 30 years ago (Chamberlin and Berg 1964). The number of examples of reiterative transcription at the start (frequently at mutant promoters), within, and at the end of bacterial, phage, and viral genes has increased rapidly in recent years (Jacques and Kolakofsky 1991, and references therein; Xiong and Reznikoff 1993). The reiterative transcription characterized in this paper closely resembles pseudotemplated transcription described previously at a phage P22 mutant sar promoter (Jacques and Susskind 1990). Although the exact mechanism of reiterative transcription remains to be established, it appears to require slippage between a homopolymeric stretch of nascent transcript and a stretch of $\langle\geqslant 3|$ complementary nucleotides in the DNA template. Most examples of reiterative transcription involve the addition of either uridines or adenines to the nascent transcript. The addition of uridines appears to occur more extensively and efficiently than the addition of the other bases. These results suggest that weak base-pairing between the transcript and template, particularly when it involves very weak rU-dA base pairs (Martin and Tinoco 1980), greatly facilitates reiterative transcription.

According to these observations, we propose the following model for reiterative transcription at the pyrBI promoter. After the synthesis of the nascent transcript AAUUU, weak base-pairing between UUU in the transcript and AAA in the DNA template allows rapid and reversible slippage between the two strands. This slippage can result in a 1- or perhaps 2-nucleotide shift in relative strand position. When UTP levels are high and slippage results in an upstream displacement of the nascent transcript, the AAA sequence in the DNA template directs the addition of another uridine residue at the $3^{\prime}$ end of the transcript. When this process occurs repeatedly, long runs of uridines are introduced into the transcript. When UTP levels are low, slippage and correct repositioning can occur without uridine addition, which provides an opportunity for the addition of a guanine residue at the $3^{\prime}$ end of the nascent transcript AAUUU. Once this addition occurs, more stable basepairing between the transcript and template prevents further slippage. This transcript is either released from the transcriptional initiation complex as an aborted transcript or extended by the addition of a cytidine residue, which apparently commits the transcriptional complex to the elongational mode. The frequency of this nonreiterative transcription increases as the level of UTP decreases.

The pattern of ladder transcript synthesis during the early phase of reiterative transcription at the pyrBI promoter is bimodal (see Fig. 2). This complex pattern may indicate preferences for particular transcript-template alignments produced by slippage. Such preferences could be related to positioning of the 5 '-terminal AA sequence of the transcript when it is still included in the transcriptional initiation complex. Consistent with this suggestion is the fact that reiterative transcription at the phage P22 mutant sar promoter, which produces transcripts with the sequence $5^{\prime}$ UUUU $_{n}$, does not exhibit a bimodal pattern of ladder transcript synthesis (Jacques and Susskind 1990).

A key element in the proposed pyr $B I$ regulatory mechanism is that the products of reiterative RNA synthesis are nonproductive. This conclusion is based on the fact 
that heterogeneous runs of uridine residues are not found near the $5^{\prime}$ ends of attenuated pyrBI transcripts synthesized in vitro. RNase $T_{1}$ digestion of these transcripts yielded only two 5 ' oligonucleotides (ACAAUUUG and AAUUUG) corresponding to the ends of transcripts initiated at the two adenine start sites located 6 and $8 \mathrm{nu}$ cleotides downstream of the promoter -10 region. Additional evidence for this conclusion is provided by previously reported primer-extension mapping of the start sites of pyr $B I$ transcripts synthesized in vitro and in vivo. In these experiments, ladders of primer-extension products were not produced, which would have been indicative of heterogeneity in the run of uridine residues near the $5^{\prime}$ ends of the transcripts (Donahue and Turnbough 1990). The reason why nascent AAUUUU Aranscripts $_{n}$ are not extended productively is not clear, but it presumably involves the $\mathrm{UUUU}_{n}$ sequence because nascent AAAAAA $_{n}$ transcripts synthesized at the $p y r B p 489$ promoter are extended into downstream pyrBI sequences. The UUUU ${ }_{n}$ sequence does not appear to be sufficient to prevent productive transcription, however, because UTP-induced reiterative transcription at a mutant lac promoter can switch efficiently to the elongational mode (Xiong and Reznikoff 1993). From the time course of ladder transcript synthesis at the pyrBI promoter in vitro, it appears that the AAUUUU $_{n}$ transcripts are released from the transcriptional initiation complex with a certain (but not always equal) probability of release after every uridine addition.

The characterization of ladder and attenuated pyrBI transcripts synthesized in vitro also demonstrates the important role of context of the UUU sequence in reiterative transcription. Transcripts initiated at the first adenine start site, which begin with the sequence ACAAUUU, do not participate in reiterative RNA synthesis. One explanation for this response is that the $\mathrm{AC}$ residues at the $5^{\prime}$ end of the transcript permit stable basepairing between the nascent transcript and DNA template, which precludes slippage and reiterative transcription. This explanation presumes that an 8- to 12-bp hybrid between the nascent transcript and DNA template forms during transcription, which remains a disputed feature of the transcription complex (Chan and Landick 1994|. Transcriptional initiation at the first adenine start site presumably does not play a major role in attenuation-independent regulation because initiation at this site occurs only at very low levels in cells grown under conditions of pyrimidine excess or limitation (Donahue and Turnbough 1990).

Why are there two mechanisms for pyrimidine-mediated regulation of pyrBI expression? A likely reason is that the mechanisms respond to different conditions of pyrimidine availability, which produce different intracellular UTP concentrations. Reiterative transcription at the pyrBI promoter is stimulated by increasing UTP concentrations up to nearly $1 \mathrm{~mm}$. Transcriptional pausing in the $p y r B I$ leader region, the sensor in attenuation control, appears to be regulated primarily between 20 and $200 \mu \mathrm{M}$ UTP (Donahue and Turnbough 1994). These results suggest that regulation by reiterative transcription functions as a response to small reductions in pyrimidine availability, whereas attenuation control serves as a response to more severe pyrimidine limitation. Such two-tiered regulation has been described for other genes; for example, repression and attenuation are used to control transcription of the $E$. coli trp operon in response to different degrees of tryptophan deprivation (Yanofsky et al. 1984).

Although there are numerous examples of reiterative transcription, some of which are required for particular protein synthesis (Jacques and Kolakofsky 1991), the pyrBI control mechanism described in this paper apparently represents the first clear case in which reiterative transcription is modulated to achieve physiologically relevant regulation of gene expression. It is unlikely that this type of regulation is limited to the pyrBI operon. Inspection of $E$. coli promoter sequences reveals that many promoters, primarily in operons involved in nucleotide and nucleic acid metabolism, contain three or more consecutive T-A base pairs near the beginning of the initially transcribed region (e.g., $\operatorname{cod} B A$, upp, $\operatorname{car} A B$, pyrF, $\operatorname{drp} A$, atpI, purMN, cya, and pheST). In some cases, expression of the operon is known to be regulated by a pyrimidine nucleotide (e.g., $\operatorname{cod} B A$, upp, $\operatorname{car} A B$, and pyrF) (Neuhard and Nygaard 1987). In the cases of the $\operatorname{cod} B A$ operon and the $p y r F$ gene, we have recently shown that high UTP levels induce strong reiterative transcription at both promoters. In addition, a mutation that prevents reiterative transcription at the $\operatorname{cod} B A$ promoter virtually eliminates pyrimidine-mediated regulation of $\operatorname{cod} B A$ expression (F. Qi and C. Turnbough, unpubl.). We anticipate that UTP-induced reiterative transcription controls the expression of many of the operons listed above, and that similar processes are involved in nucleotide-mediated regulation of a wide range of other bacterial and perhaps eukaryotic genes.

\section{Materials and methods}

Bacterial strains

E. coli $\mathrm{K}-12$ strains CLT42 [F ${ }^{-}$car-94 $\Delta$ (argF-lac) U169 rpsL150 thiA1 relA1 deoC1 ptsF25 flbB5301 rbsR] and CLT71 [CLT42 $\triangle$ pyrBa483) were described previously (Liu and Turnbough 1989). Strains CLT214 and CLT235 are isogenic with strains CLT42 and CLT71, respectively, except that they carry the pyrBI promoter mutation pyrBp488. Strains CLT214 and CLT235 were constructed by allelic replacement of the pyrBI promoter region by use of homologous recombination and subsequent bacteriophage Pl-mediated transductions to generate the desired genetic backgrounds essentially as described for the construction of strain CLT71 (Liu and Turnbough 1989). The presence of the correct mutant pyrBI promoter-leader regions in strains CLT214 and CLT235 was confirmed by PCR amplification of these regions in the chromosome (Dorit and Ohara 1991) followed by determination of their DNA sequences with the Sequenase kit (U.S. Biochemical). The absence of large deletions, insertions, or rearrangements near pyrBI in these strains was shown by Southern hybridization as described previously (Liu and Turnbough 1989). 
DNA preparations, restriction digests, ligations, transformations, and oligonucleotide-directed mutagenesis

Plasmid DNA was isolated by use of Qiagen kits, and chromosomal DNA was prepared as described previously (Liu and Turnbough 1994). Restriction digests, ligations, and transformations were performed as described previously (Roland et al. 1985). The oligonucleotide-directed mutagenesis procedures used to construct the pyrBp488 (Kunkel et al. 1987) and pyrBp489 (Liu and Turnbough 1989) mutations were essentially as described previously, and the mutations were verified by DNA sequence analysis.

\section{Media and culture methods}

Cells used for ATCase assays were grown at $37^{\circ} \mathrm{C}$ in $\mathrm{N}^{-} \mathrm{C}^{-}$ medium (Alper and Ames 1978) supplemented with $10 \mathrm{~mm}$ $\mathrm{NH}_{4} \mathrm{Cl}, 0.4 \%$ glucose, $0.015 \mathrm{~mm}$ thiamine- $\mathrm{HCl}, 1 \mathrm{~mm}$ arginine, and either $1 \mathrm{~mm}$ uracil or $0.25 \mathrm{mM}$ UMP. Cell growth was monitored as described previously (Liu et al. 1993).

\section{ATCase assay}

Cells from exponential-phase cultures were used to measure ATCase activities as described previously (Liu et al. 1993).

\section{In vitro transcription}

DNA templates and purified RNA polymerase holoenzyme containing $\sigma^{70}$ were prepared as described previously (Turnbough et al. 1983). The DNA templates were 758-bp PvuII fragments containing the pyrBI promoter and downstream sequences through the leader region and first 46 codons of the pyrB gene. Transcription reaction mixtures $(10 \mu \mathrm{l})$ contained $20 \mathrm{~mm}$ Tris- $\mathrm{HCl}$ (pH 7.9); $10 \mathrm{mM} \mathrm{MgCl}_{2} ; 50 \mathrm{~mm} \mathrm{KCl} ; 0.2 \mathrm{~mm} \mathrm{Na}$ EDTA; $_{0} 0.1 \mathrm{~mm}$ dithiothreitol; $200 \mu \mathrm{M}$ each of ATP, GTP, CTP, and UTP, unless indicated otherwise; $10 \mathrm{nM}$ DNA template; and $100 \mathrm{nM}$ RNA polymerase (active enzyme, 20\%). Radiolabeled nucleoside triphosphates (typically $2 \mathrm{Ci} / \mathrm{mmole}$, Amersham) were included in the mixtures as indicated in the text. Reactions were initiated by addition of RNA polymerase and incubated at $37^{\circ} \mathrm{C}$ for $30 \mathrm{~min}$, unless indicated otherwise. Reactions were stopped by addition of $10 \mu \mathrm{l}$ of a solution containing $7 \mathrm{M}$ urea, $2 \mathrm{mM}$ $\mathrm{Na}_{2}$ EDTA, and $0.025 \%$ each of bromophenol blue and xylene cyanol. An equal volume was removed from each mixture for analysis. Transcripts were separated on a polyacrylamide sequencing gel containing $7 \mathrm{M}$ urea (Liu and Turnbough 1989) and visualized by autoradiography. Transcripts were quantitated by scanning of gels with a Molecular Dynamics PhosphorImager or by excision of bands from gels and measurement of radioactivity in a scintillation counter.

\section{Acknowledgments}

C.L. and L.S.H. contributed equally to this work. We thank Melissa Caimano for constructing the original version of the pyrBp488 mutation and Fengxia Qi for assistance in preparing figures. This work was supported by National Institutes of Health grant GM29466.

The publication costs of this article were defrayed in part by payment of page charges. This article must therefore be hereby marked "advertisement" in accordance with 18 USC section 1734 solely to indicate this fact.

\section{References}

Alper, M.D. and B.N. Ames. 1978. Transport of antibiotics and metabolite analogs by systems under cyclic AMP control: Positive selection of Salmonella typhimurium cya and crp mutants. J. Bacteriol. 133: 149-157.

Andersen, J.T., K.F. Jensen, and P. Poulsen. 1991. Role of transcription pausing in the control of the pyrE attenuator in Escherichia coli. Mol. Microbiol. 5: 327-333.

Chamberlin, M. and P. Berg. 1964. Mechanism of RNA polymerase action: Characterization of the DNA-dependent synthesis of polyadenylic acid. J. Mol. Biol. 8: 708-726.

Chan, C.L. and R. Landick. 1994. New perspectives on RNA chain elongation and termination by $E$. coli RNA polymerase. In Transcription: Mechanisms and regulation (ed. R. Conaway and J. Conawayl, pp. 297-320. Raven Press, New York.

Donahue, J.P. and C.L. Turnbough Jr. 1990. Characterization of transcriptional initiation from promoters $P_{1}$ and $P_{2}$ of the pyrBI operon of Escherichia coli K12. I. Biol. Chem. 265: 19091-19099.

1994. Nucleotide-specific transcriptional pausing in the pyrBI leader region of Escherichia coli K-12. J. Biol. Chem. 269: 18185-18191.

Dorit, R.L. and O. Ohara. 1991. Direct DNA sequencing of PCR products. In Current protocols in molecular biology led. F.M. Ausubel, R. Brent, R.E. Kingston, D.D. Moore, J.G. Seidman, J.A. Smith, and K. Struhl), pp. 15.2.3. John Wiley \& Sons, New York.

Jacques, J.-P. and D. Kolakofsky. 1991. Pseudo-templated transcription in prokaryotic and eukaryotic organisms. Genes \& Dev. 5: 707-713.

Jacques, J.-P. and M.M. Susskind. 1990. Pseudo-templated transcription by Escherichia coli RNA polymerase at a mutant promoter. Genes \& Dev. 4: 1801-1810.

Jin, D.J. and C.L. Turnbough Jr. 1994. An Escherichia coli RNA polymerase defective in transcription due to its overproduction of abortive initiation products. $/$. Mol. Biol. 236: 72-80.

Kunkel, T.A., J.D. Roberts, and R.A. Zakour. 1987. Rapid and efficient site-specific mutagenesis without phenotypic selection. Methods Enzymol. 154: 367-382.

Landick, R. and C.L. Turnbough Jr. 1992. Transcriptional attenuation. In Transcriptional regulation (ed. S.L. McKnight and K.R. Yamamoto), pp. 407-446. Cold Spring Harbor Laboratory Press, Cold Spring Harbor, New York.

Liu, C. and C.L. Turnbough Jr. 1989. Multiple control mechanisms for pyrimidine-mediated regulation of pyrBI operon expression in Escherichia coli K-12. J. Bacteriol. 171: 33373342.

Liu, J. and C.L. Turnbough Jr. 1994. Effects of transcriptional start site sequence and position on nucleotide-sensitive selection of alternative start sites at the pyrC promoter in Escherichia coli. J. Bacteriol. 176: 2938-2945.

Liu, C., J.P. Donahue, L.S. Heath, and C.L. Turnbough Jr. 1993. Genetic evidence that promoter $\mathrm{P}_{2}$ is the physiologically significant promoter for the pyrBI operon of Escherichia coli K-12. J. Bacteriol. 175: 2363-2369.

Martin, F.H. and I. Tinoco Jr. 1980. DNA-RNA hybrid duplexes containing oligo(dA:rU) sequences are exceptionally unstable and may facilitate termination of transcription. Nucleic Acids Res. 8: 2295-2299.

Neuhard, J. and P. Nygaard. 1987. Purines and pyrimidines. In Escherichia coli and Salmonella typhimurium: Cellular and molecular biology (ed. F.C. Neidhardt, J.L. Ingraham, K.B. Low, B. Magasanik, M. Schaechter, and H.E. Umbarger), pp. 445473. American Society for Microbiology, Washington, D.C. 
Roland, K.L., F.E. Powell, and C.L. Turnbough Jr. 1985. Role of translation and attenuation in the control of $p y r B I$ operon expression in Escherichia coli K-12. J. Bacteriol. 163: 991999.

Roland, K.L., C. Liu, and C.L. Turnbough Jr. 1988. Role of the ribosome in suppressing transcriptional termination at the pyrBI attenuator of Escherichia coli K-12. Proc, Natl. Acad. Sci. 85: 7149-7153.

Turnbough, C.L. Jr. 1983. Regulation of Escherichia coli aspartate transcarbamylase synthesis by guanosine tetraphosphate and pyrimidine ribonucleoside triphosphates. I. Bacteriol. 153: 998-1007.

Turnbough, C.L. Jr., K.L. Hicks, and J.P. Donahue. 1983. Attenuation control of pyrBI operon expression in Escherichia coli K-12. Proc. Natl. Acad. Sci. 80: 368-372.

Xiong, X.F. and W.S. Reznikoff. 1993. Transcriptional slippage during the transcription initiation process at a mutant lac promoter in vivo. J. Mol. Biol. 231: 569-580.

Yanofsky, C., R.L. Kelley, and V. Horn. 1984. Repression is relieved before attenuation in the trp operon of Escherichia coli as tryptophan starvation becomes increasingly severe. $I$. Bacteriol. 158: 1018-1024. 


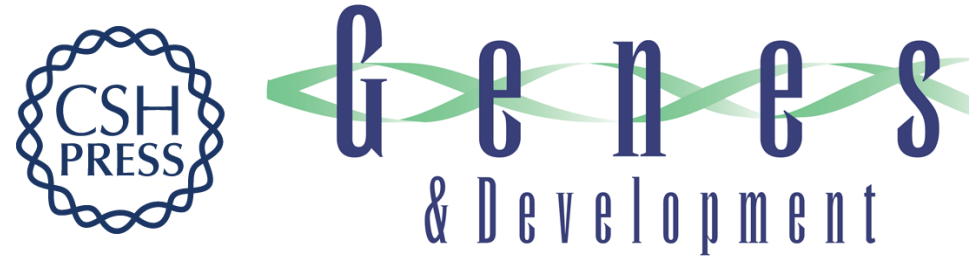

\section{Regulation of pyrBI operon expression in Escherichia coli by UTP-sensitive reiterative RNA synthesis during transcriptional initiation.}

C Liu, L S Heath and C L Turnbough

Genes Dev. 1994, 8:

Access the most recent version at doi:10.1101/gad.8.23.2904

References This article cites 19 articles, 13 of which can be accessed free at:

http://genesdev.cshlp.org/content/8/23/2904.full.html\#ref-list-1

License

Email Alerting Receive free email alerts when new articles cite this article - sign up in the box at the top Service right corner of the article or click here.

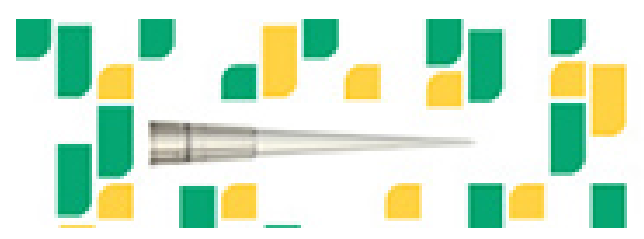

Focused on your science. 